http://jmscr.igmpublication.org/home/ ISSN (e)-2347-176x ISSN (p) 2455-0450 crossref DOI: https://dx.doi.org/10.18535/jmscr/v9i7.14

\title{
Prevalence of MDR Pathogens in Urine of Paediatric Patients in a Tertiary Care Hospital
}

\author{
Authors \\ Munish Rastogi', Mohit Kumar ${ }^{2}$ \\ ${ }^{1}$ Assistant Professor U.I. H.S. CSJMU Kanpur University \\ ${ }^{2}$ Junior Resident GMC Kannauj \\ *Corresponding Author \\ Prof. Dr Dolly Rastogi \\ Head of Department Physiology GMC Kannauj
}

\begin{abstract}
Introduction: Nowadays, antimicrobial resistance is a challenge faced by physicians globally. Infections due to Multidrug Resistant Organisms (MDROs) is a significant problem in Paediatric Intensive Care Unit (PICU).
\end{abstract}

Aim: To identify the burden of antimicrobial resistance in hospital isolates from paediatric patients and elucidate the resistance pattern of MDROs.

Material and Methods: All the clinical specimen received from pediatric outpatient and inpatient were included in the study and data were collected and processed on blood agar, chocolate agar and MacConkey agar. Cled agar from those yielding growth on urine culture. The bacterial isolates were identified with standard bio-chemical tests. Antibiotic susceptibility testing was done according to CLSI guidelines 2019 by Kirby Bauer disk diffusion method.

Results: A total of 100 (39.07\%) bacteria were isolated from 256 clinical samples, 65 bisolates were from urine followed by blood (22), exudates (9) and respiratory (4) samples. 22 were MDROs, 14 were from urine, blood(5), exudates(2) and respiratory(1) samples respectively. Isolates of E. coli (54\%), Klebsiella spp (36\%). and Enterococcus (0.9\%) spp. were the common MDROs. The most sensitive antibiotic for gram negative bacteria was polymyxin-B (100\%) followed by imipenem (98\%) and meropenem (90\%). And the most sensitive antibiotic for gram positive bacteria was Linezolid (100\%) Vancomycin (97\%) followed by Teicoplanin (94\%).

Conclusion: This study reveals significant prevalence of MDR pathogens among paediatric population. E. coli, Klebsiella spp. and Enterococcus spp. were the common MDROs encountered in the study, mostly isolated from urine culture.

Keywords: Bacterial, Drug resistance, Escherichia coli, Urinary tract infection.

\section{Introduction}

Antimicrobial resistance is on the rise, creeping into the paediatric population too. Infections due to MDROs is a significant problem in paediatric ICU. In developing countries, MDROs causing neonatal infections are increasing and this has been attributed to the production of Extended Spectrum Beta Lactamases (ESBLs), Amp C $\beta$ lactamases, carbapenemases and aminoglycoside modifying enzyme ${ }^{[1]}$. Sepsis due to MDROs is a significant problem worldwide ${ }^{[2]}$ Klebsiella pneumoniae and Staphylococcus aureus have been 
identified as the most common cause for neonatal sepsis in India. Prolonged hospitalisation, increased use of antimicrobials and vulnerability are some of the factors that favour neonatal colonisation with MDROs ${ }^{[3,4]}$. It has also been observed that MDROs causing Urinary Tract Infections (UTI) in the paediatric age group include mainly E. coli and Klebsiella $\mathrm{spp}^{[5]}$. Infections due to Multidrug-Resistance (MDR) Gram negative organisms are on the rise globally ${ }^{[6,7]}$. Although the most common pathogen in the NICU is Gram positive coccus, the incidence of bacteremia caused by Gram-negative bacilli (GNB) has increased in the past decades, and GNB bacteremia is often associated with a higher mortality rate ${ }^{[8,9,10]}$. The emergence of multidrug resistance (MDR) among these organisms deserves particular concern, because treatment options of antimicrobial agents for an MDR strain are often limited and inappropriate initial antibiotics will predispose these neonates to an especially high risk of severe sepsis and a poor outcome ${ }^{[11,12]}$.

\section{Material and Method \\ Material}

256 Sample from paediatric patient will be collected. from inpatients admitted to the ICU and NICU were collected from Rama Medical college hospital and research centre study was conducted from January 2019 to December 2019.

\section{Method}

All the sample was collected from pediatric outpatient and inpatient were included and the study and data were collected in MacConkey agar Cled agar from those yielding growth on culture. The bacterial isolate was identified with standard bio- chemical test.

Preliminary identification was done with the help of the following methods:- Gram Stain:- The suspected colonies were stained using gram stain method and their shape, colour and arrangement were observed under light microscope.

Procedure of Gram Staining: -Gram Staining is a differential staining and it consist of four steps:
Primary stain (crystal violet) for 1 minute. Mordant (gram,s iodine) for 1 minute. Decolourizer is - (acetone) for $5 \mathrm{sec}$. Counter stain (safranin) for $30 \mathrm{~s}$

Inclusion Criteria: Paediatric patients with clinical infection and sample, Pus, Urine, Blood, Body fluid will be collected. Social demographic date, medical history, clinical history regarding age, gender type of paediatric patient percentage and antibiotic therapy will be taken.

Exclusion Criteria: Repeat isolates from the same patients and from the same site/specimen all excluded.

\section{Biochemical Tests Catalase Test Principle -}

This test demonstrates the presence of catalase an enzymes that catalyses the release of oxygen from hydrogen peroxide.

Procedure- Take a clean grease free slide. Divide it into two halves. One half serves at test and the other as control. Put one drop of 3\% H202 each on the test and control halves Now, with the help of wooden stick a separate colony from the culture plate and touch on the surface of $\mathrm{H} 2 \mathrm{O} 2$ marked as test. Look for the effervescence production. P.CATCC Staphylococcus aurous (25923) N.CATCC Enterococcus faecal is (29212)

\section{Statistical Analysis}

Statistical analysis was performed by using computer-based software, Statistical Package for Social Science (SPSS). Mean values of parameters were compared to determine.

\section{Observation \& Result}

In out of 256 total 100 sample were found with various bacterial growth which mean finding of bacteria was $39.88 \%$ in our study. In which the finding of different type of bacteria was E.coli (18.35\%), Klebsiella spp (7.42\%) Pseudomonas aeruginosa (5.46\%), CONS (5.46\%), Enterococcus spp (14.84\%), Stapylococcus aureus $(7.03 \%)$. 


\section{JMSCR Vol||09||Issue||07||Page 72-78||July}

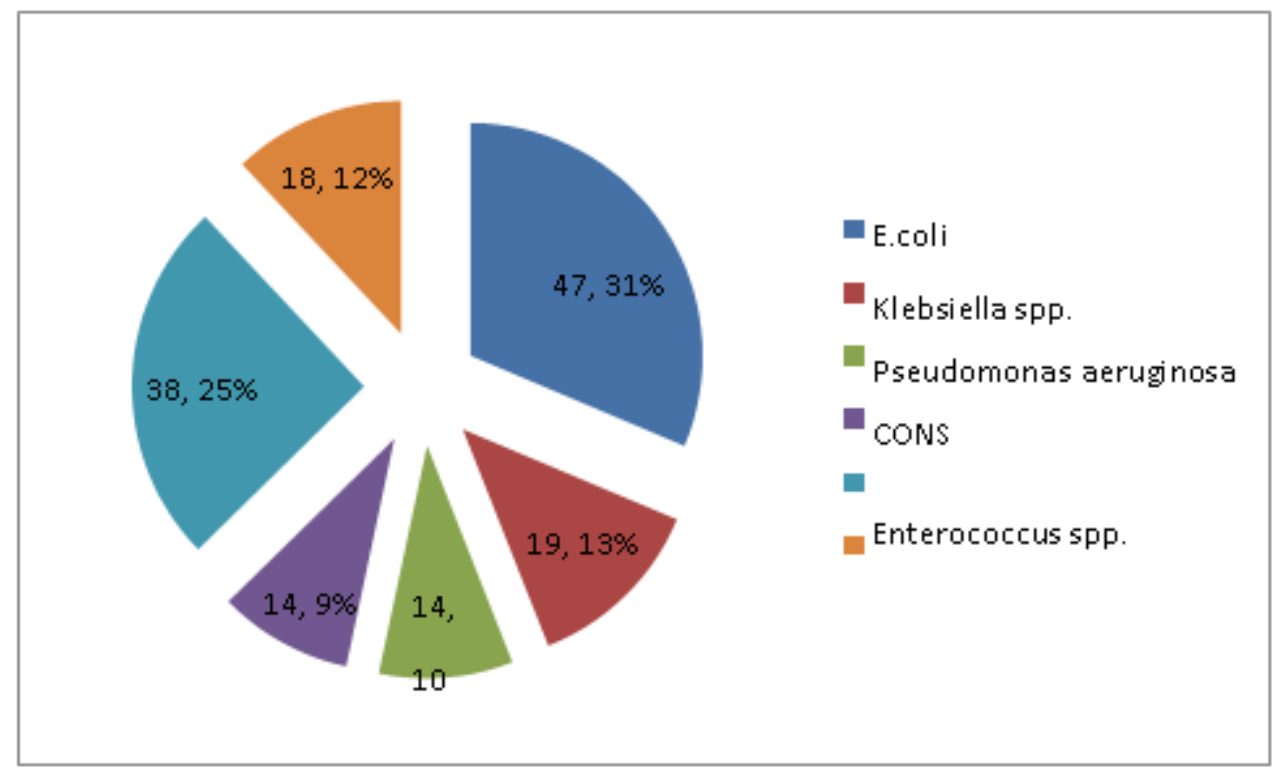

Fig No 1 Number of different bacteria found in total 150 sample

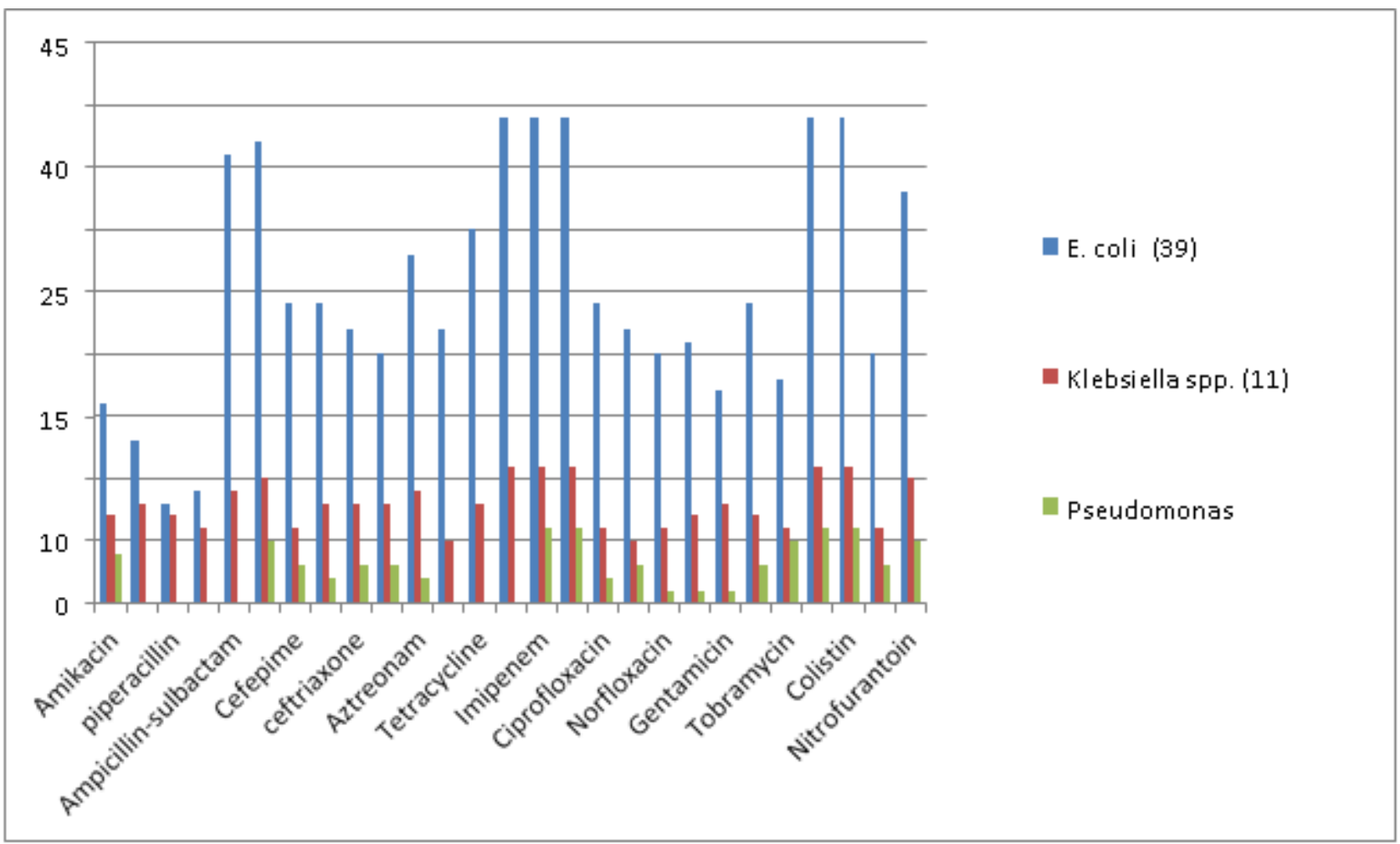

Fig No 2 bar chart showing antibiotic sensitivity for gram negative cocci

In case of E.coli, Polymyxin-B, colistin, Imipenem, Meropenem, Tigecycline, showed $100 \%$ Sensitivity followed by Amplicillin sulbactam (83\%), Piperacillin tazobactam (93\%), Nitrofurantion $(93.25 \%)$.

In case of Klebsiella spp., Polymyxin-B, colistin, Imipenem, Meropenem, Tigecycline, showed
$100 \%$ Sensitivity followed by Amplicillin sulbactam (83\%), Piperacillin tazobactam (93\%), Nitrofurantion (93.25\%), Netilmycin (75\%).

In case of Pseudomonas aeruginosa, PolymyxinB, colistin, Imipenem, Meropenem, showed 100\% Sensitivity followed b, Piperacillin tazobactam (93\%), Nitrofurantion (93.25\%). 


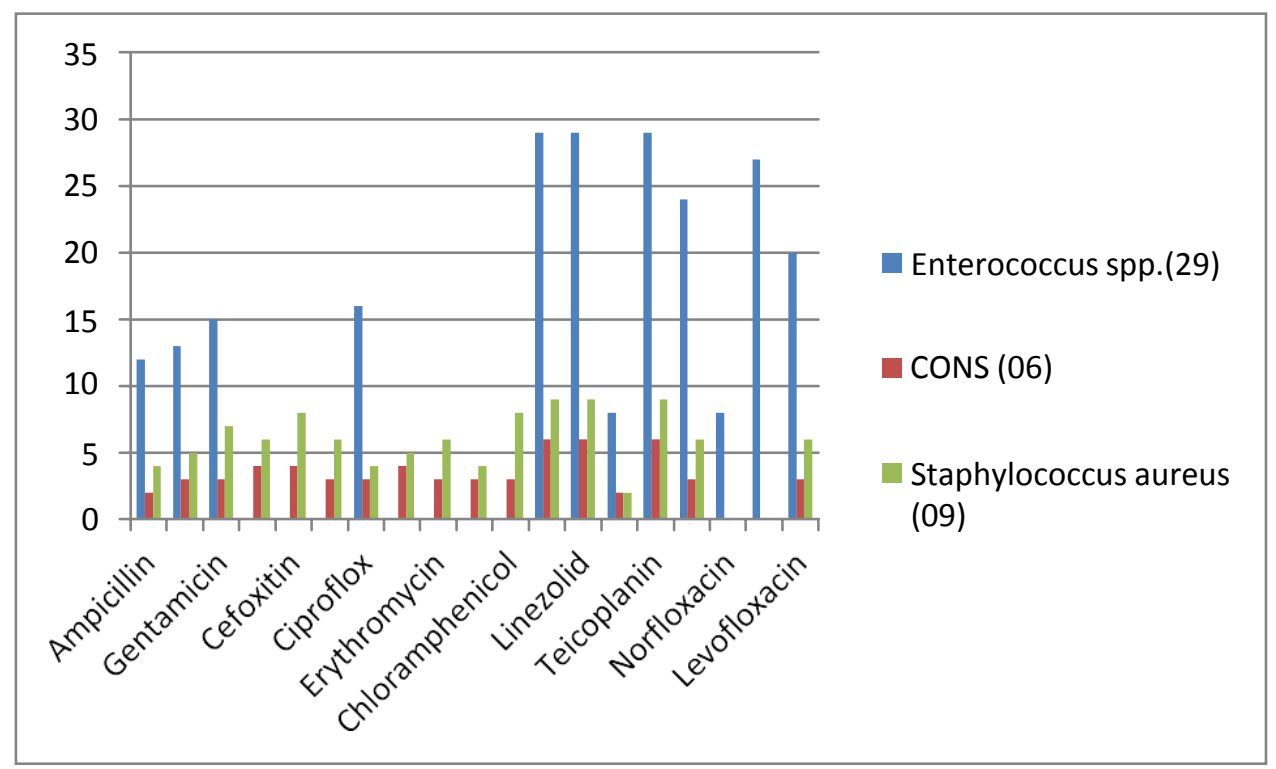

Fig 3 showing the antibiotic sensitive for gram positive coci

In case of Enterococcus spp., Vancomycin Linezolid, Teicoplanin showed 100\% sensitivity followed by, Tetracycline (75\%), Nitrofurantoin (75\%). In case of CONS, Vancomycin Linezolid, Teicoplanin showed $(100 \%)$ Sensitivity followed by, Tetracycline (75\%), Cefoxitin (80\%) \& Levofloxcin (75\%). In case of Staphylococcus aureus, Vancomycin Linezolid, Teicoplanin showed (100\%) sensitivity followed by, Tetracycline (40 \%), Cefoxitin (50\%) \& Levofloxcin (40\%), Gentamicin (60\%).

\section{Discussion}

In the study done by Durgesh et al7, Escherichia coli was the predominant $(31.25 \%)$ isolates causing UTI, followed by Staphylococcus aureus (25\%), Pseudomonas aeruginosa (15.62\%), Proteus mirabilis (15.62\%), Klebsiella pneumoniae $(6.25 \%)$ and Serratia marcescens $(6.25 \%)$ where as in our study the most common organism causing UTI was Escherichia coli $(15.23 \%)$ isolates followed by Enterococcus spp. (11.23\%), Klebsiella sppecies (4.29), Staphylococcus aureus (3.51\%), Pseudomonas aeruginosa (2.34\%), CONS (2.34).

Similarly, in the study done by Yolbaset al6 and Mostafa et $\mathrm{al}^{8}$ and, Escherichia coli was the most common organism causing urinary tract infection followed by Klebsiella which was similar to our study. Similar results were observed in studies done by Rehamanet al9 and CWkwan et al ${ }^{10}$. This difference may be due to the variation in geographical distribution.

Study done by Haller et al showed the effective empirical intravenous and oral antibiotics for the treatment of community-acquired UTIs include Ampicillin and Aminoglycosides ${ }^{11}$, whereas the oral antibiotic of choice by Prais D et $\mathrm{al}^{12}$ and Hoberman et $\mathrm{al}^{13}$ showed AmoxicillinClavulanate or Cefuroxime and Cefixime respectively.

Compared to the study done by Yolbas et al6, in which Escherichia coli was resistant to Amikacin in 3\%, Nitrofurantoin 9\%, Trimethoprim/ Sulfamethoxazole $58 \%$ and Cefotaxime $51 \%$, in our study Escherichia coli showed more resistant pattern to these antibiotics i.e Amikacin (15.6\%), Nitrofurantoin (46.8\%), Cotrimoxazole (68.75\%) and Cefotaxim ( $81 \%)$. The reason for increase in resistance may be due to the excessive use of third generation Cephalosporins both as oral and intravenous route.

In the study done by Durgesh et $\mathrm{al}^{7}$, the mean sensitivity to Penicillin and Ciprofloxacin were $70.83 \%$ and $60 \%$ respectively. Staphylococcus aureus showed $75 \%$ resistance to Methicillin, Oxacillin and Vancomycin. Uropathogens were sensitive to Norfloxacin, Co- trimoxazole and 
Ofloxacin. These results were in contrast to our study in which Escherchia coli was sensitive to Amoxycillin and Ciprofloxacin in only $6 \%$ and $15.6 \%$ respectively, and in case of Klebsiella pneumoniae none was sensitive to Amoxycillin and only $40 \%$ were sensitive to Ciprofloxacin. The reason for this difference may be due to the injudicious over the counter use of antibiotics.

In the study done by Mostafa et $\mathrm{al}^{8}$, Escherichia coli had a sensitivity rate of $97.8 \%$ to Ceftriaxone and $95.2 \%$ to Cefotaxime in contrast to our study in which Cefotaxim was sensitive only in $18.7 \%$ of cases. The highest resistance rate of Escherichia coli was to Penicillin (95.2\%) followed by Amoxycillin and Cotrimoxazole (79 and $74.2 \%$ respectively) in the study by Mostafaet al11 whereas in our study Escherichia coli showed high resistant to Amoxycillin (93\%) followed by Nalidixic acid (87.5\%). According to Mostafaet al8 Klebsiella spp. showed the highest sensitivity to Ciprofloxacin (95.1) and Ceftriaxone 90.7\% which was in contrast to our study in which Klebsiella was sensitive to Ciprofloxacin and Cefotaxim only in $40 \%$ and $30 \%$ respectively. in our study Klebsiella showed highest resistant to Amoxycillin which was similar to study done by Mostafa et $\mathrm{al}^{8}$.

In Study done by Reham et $\mathrm{al}^{9}, 59.9 \%$ isolates of Escherichia coli were multidrug resistant where as in our study only one Escherichia coli was multidrug resistant. In our study Escherichia coli showed increase in resistant to oral antibiotics; Amoxycillin (93\%), Ofloxacin (81.2\%), Ciprofloxacin $(84.3 \%)$ Cotrimoxazole (68.75\%), and Nitrofurantoin $(46.8 \%)$ which was similar to the results in study done by Reham et $\mathrm{al}^{9}$. The reason may be easy access to oral antibiotics from pharmacy.

In our study E. coli was sensitive to Cefotaxim in only $18.75 \%$ where as in study done by $\mathrm{CW}$ Kwan et al10, Escherichia coli was sensitive to greater than $95 \%$ of third-generation Cephalosporins (Ceftriaxone and Ceftazidime). In their study bacteria were frequently resistant to ampicillin (54.4\%) and Trimethoprim-
Sulfamethoxazole (40.4\%) which was comparable to our study.

In our study Escherichia coli (81.2\%) and Klebsiella pneumoniae (70\%) showed increase in resistance pattern to Cephalosporins which was comparable to study done by Stephanie A et al14 in which there was high rate of resistance to third generation Cephalosporins in subpopulations of children admitted to the hospital for UTIs.

In the study done by Rasoulet al15, most isolates showed high resistance against Ampicillin, Cotrimoxazole, Nalidixic acid, and Nitrofurantoin and Klebsiella isolates showed more resistance against tested antibiotics than Escherichia coli isolates which was comparable to our study.

Although our study suggested high resistance to oral antibiotics, there was a caveat that this study included in-patients only. This might have caused some selection bias. However, this information raises an important issue regarding antibiotic resistance in UTI. A future study including both in-patients and out-patients would help clarify if resistance to oral antibiotics has indeed emerged in the organisms causing UTI in the community.

\section{Conclusion}

This study reveals significant prevalence of MDR pathogens among paediatric population ESBL (E.coli), MBL (P.aeruginosa), MRSA (Staphylococcus aureus) \& VRE (Enterococcus spp.). E. coli, Klebsiella spp. and Enterococcus spp. were the common MDROs encountered in the study, most of the isolates were from urine culture. MDROs are present among outpatients as well, warranting judicious use of antibiotics and adequate infection control measures to prevent spread of these potential pathogens.

Knowledge of the antibiotics susceptibility pattern of the isolates from paediatric patient infection is crucil for planning the appropriate treatment of these cases prior to getting the susceptibility reports from the laboratory 


\section{Reference}

1. Gajul SV, Mohite ST, Mangalgi SS, Wavare SM, Kakade SV. Klebsiella pneumoniae in septicemic neonates with special reference to extended spectrum $\beta$ lactamase, Amp C, metallo $\beta$-lactamase production and multiple drug resistance in tertiary care hospital. J Lab Physicians. 2015;7(1):32-37

2. Muley VA, Ghadage DP, Bhore AV. Bacteriological profile of neonatal septicemia in a tertiary care hospital from Western India. J Global Infect Dis. 2015;7(2):75-77.

3. De Oliveira Costa P, Atta EH, da Silva AR. Infect with multidrug-resistant gramnegative bacteria in a pediatric oncology intensive care unit: risk factors and outcomes. J Pediatr (Rio J). 2015;91 (5):435-41

4. Arnold, J.C.; Bradley, J.S. Osteoarticular Infections in Children. Infect. Dis. Clin. 2015, 29, 557-574.

5. Folgori L, Livadiotti S, Carletti M, Bielicki J, Pontrelli G, DegliAtti ML, et al. Epidemiology and clinical outcomes of multidrug-resistant Gram-negative bloodstream infections in a European tertiary pediatric hospital during a 12 month period. Pediatr Infect Dis J. 2014;33(9):929-32

6. Siddiqui N, Qamar FN, Jurair H, Haque A. Multi-drug resistant gram-negative infections and use of intravenous polymyxin B in critically ill children of developing country: retrospective cohort study. BMC Infectious Diseases. 2014; $14: 626$

7. Benner KW, Parbhakaran P, Lowros AS. Epidemiology of infections due to extended spectrum beta-lactamaseproducing bacteria in a pediatric intensive care unit. J Pediatr Pharmacol Ther. 2014;19(2):83-90

8. Gyawali N, Sanjana RK. Bacteriological profile and antibiogram of neonatal septicemia. Indian $\mathbf{J}$ Pediatr. 2013;80(5):371-74.

9. Patel G, Bonomo RA. "Stormy waters ahead": global emergence of carbapenemases. Front Microbiol 2013; 4:1-17.

10. Cornaglia G, Giamarellou H, Rossolini GM. Metallo- $\beta$-lactamases: a last frontier for $\beta$-lactams? Lancet Infect Dis 2011; 11:381-93.

11. Taneja N, Chatterjee SS, Singh M, Singh S, Sharma M. Pediatric urinary tract infections in a tertary care center from North India. Indian J Med Res. 2010;131:101-05.

12. DeLeo, F.R.; Otto, M.; Kreiswirth, B.N.; Chambers, H.F. Community- associated Methicillin-resistant Staphylococcusaureus. Lancet 2010, 375, 1557-1568.

13. I. Yolbas, R. Tekin1, S. Kelekci, A. Tekin, M.H. Okur, A. Ece, A. Gunes, V. Sen. Community-acquired urinary tract infections in children: pathogens, antibiotic susceptibility and seasonal changes. European Rev Med Pharmacol Sci 2013;17:971-976.

14. Durgesh D. Wasnik, P. M. Tumane. Prevalence and antibacterial susceptibility pattern of Urinary Tract Infection Causing Human Pathogenic Bacteria. Asian J Biomed Pharmaceutical Sci 2012;2:1-3.

15. Mostafa Sharifi an, Abdollah Karimi, Sedigheh Rafi ee, Tabatabaei and Navid Anvaripour. Microbial Sensitivity Pattern in Urinary Tract Infections in Children:A Single Centre Experience of 1,177 Urine Cultures. J Infect Dis 2006;59:380- 382.

16. RehamIssa Al-Mardeni, Adel Batarseh, Lina Omanish, Majdolin Shraideh, Basma Batarseh, Nidal Unis. Emprical Treatment for Pediatric Urinary Tract Infection and Resistance Patterns of Uropathogens, in Queen Alia Hospital and Prince A'Isha Military Centre - Jordan. Saudi J Kidney 
Dis Transplant 2009;20(1):135-139.

17. CW Kwan, H Onyett. Communityacquired urinary tract pathogens and their resistance patterns in hospitalized children in south-eastern Ontario between 2002 and 2006. Paediatr Child Health 2008; 13:759762.

18. Haller M, Brandis M, Berner R. Antibiotic resistance of urinary tract pathogens and rationale for empirical intravenous therapy. Pediatr Nephrol 2004;19:982986.

19. Prais D, Straussberg R, Avitzur Y, Nussinovitch M, Harel L, Amir J. Bacterial susceptibility to oral antibiotics in community acquired urinary tract infection. Arch Dis Child 2003;88:215218.

20. Hoberman A, Wald ER, Hickey RW, et al. Febrile children oral versus initial intravenous therapy for urinary tract infections in young. Pediatrics. 1999;104;79- 86.

21. Stephanie A. Lutter, MD; Melissa L. Currie, MD; Lindsay B. Mitz, BA; Larry A. Greenbaum, MD, PhD. Antibiotic Resistance Patterns in Children Hospitalized for Urinary Tract Infections. Arch Pediatr Adolesc Med. 2005;159:924928.

22. Rasoul Yousefi Mashouf, Hooshang Babalhavaeji and Javad Yousef. Urinary Tract Infections: Bacteriology and Antibiotic Resistance Patterns. Indian Pediatrics. 2009;46: 617-620. 